\title{
REAL STRUCTURE AND RESIDUAL STRESSES IN ADVANCED WELDS DETERMINED BY X-RAY AND NEUTRON DIFFRACTION
}

\author{
Karel Trojan $^{a, *}$, Charles Hervoches ${ }^{b}$, Kamil KolaříK ${ }^{a}$, \\ Nikolaj Ganev ${ }^{a}$, Pavol Mikula ${ }^{b}$, JiŘí ČAPeK ${ }^{a}$ \\ ${ }^{a}$ CTU in Prague, Department of Solid State Engineering, Faculty of Nuclear Sciences and Physical Engineering, \\ Trojanova 13, 12000 Prague 2, Czech Republic \\ ${ }^{b}$ Nuclear Physics Institute, ASCR, v.v.i., Department of Neutron Physics, 25068 Řě, Czech Republic \\ * corresponding author: Karel.Trojan@fjfi.cvut.cz
}

\begin{abstract}
The paper outlines the capability of X-ray diffraction (XRD) for evaluation of real structure changes and residual stresses (RS) on cross-section of advanced thick welds due to the welding of ferromagnetic plates. The results of neutron diffraction describe a three-dimensional state of RS and also verify previous assumptions of RS redistribution as a result of the surface preparation for determination 2D maps measured by XRD.
\end{abstract}

KEYWORDS: laser and MAG welding, residual stresses, X-ray diffraction, neutron diffraction.

\section{INTRODUCTION}

High local residual stresses existing in components could have vital influence on its properties. When a welded part with high local RS is machined the significant distortion may occur, due to the disruption of the equilibrium state of RS. On the other hand high tensile RS due to welding have a strong negative effect on the strength properties, especially under fatigue loading. Therefore, the determination of the $\mathrm{RS}$ distribution in complex welded components is accentuated.

Recent practical approaches distinguish between local and global welding RS [1]. The local RS are resulting from the local heating and cooling processes in the weld metal and adjacent heat affected zone (HAZ). Global RS exist in the entire component and they are resulting from shrinkage processes which occur in the whole component [1]. These global RS are affected by the stiffness of the component and the local shrinkage processes in the direction of black arrows in Fig. 1. Tensile RS around a single pass weld in a plane sample are expected due to the restraint which is generated by the cool adjacent zones in the base material of the plate [1]. Therefore, the main direction of tensile RS due to shrinkage is applied along the weld. In the perpendicular direction, the tensile RS should reach lower values according to this model.

For a complete description of the austenite phase, the transformation associated with a change in volume must be taken into consideration as a significant source of RS especially in high strength steels. The local compressive RS, which arise in the weld zone as a consequence of the restrained volume expansion during the transformation of austenite in martensite, bainite or ferrite, are superimposed to the global tensile RS

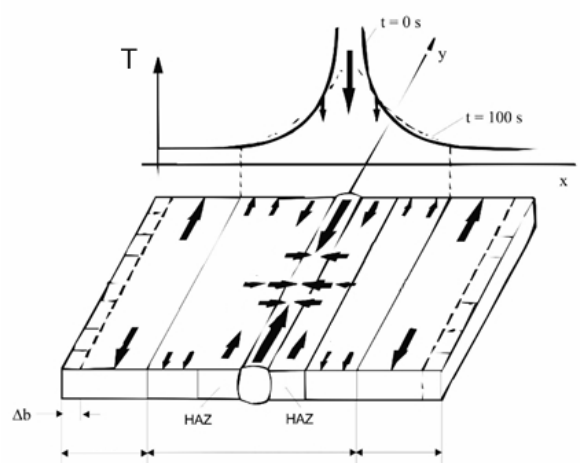

FIGURE 1. Shrinkage tendency and constraint conditions in a single pass weld (schematically) [1.

explained according to the model described above (Fig. 1] [1]. The increase in the cooling rate of the welded plates will lead to quenching and formation of local compressive RS. Laser welding supplies into the weld lower heat input so that cooling of the weld is faster compared to the MAG technology. The resulting distribution of residual stresses is a combined effect of hindered shrinkage and phase transformations.

Developed laser welding methods using high power diode lasers (HPDL) took over the capability to fill groove with cold or hot wire metal from metal active gas (MAG) welding and thus to change mechanical properties of welds reducing their hardness due to the quenching [2]. Profitable changes of real crystallographic structure and RS in comparison with conventional laser welds improve the results during impact and tensile test and mainly enhance fatigue life as a result of a favourable distribution of RS and its relaxation during welding in the weld zone and HAZ 3 . 
To verify the results obtained by XRD, where the samples were cut in two pieces, it is preferable to use the neutron diffraction. It has distinguished benefits such as the unique deep-penetration, threedimensional mapping capability, and volume-averaged bulk measurements characteristic of the scattering neutron beam for steel sample thickness up to $50 \mathrm{~mm}$ without cutting 4 .

\section{EXPERIMENTAL METHODS}

The analysed samples were prepared by a HPDL laser with cold wire and MAG welding (Metal Active Gas (MAG) welding subtype of gas metal arc welding) from two $20 \mathrm{~mm}$ thick sheets made of S355J2 steel. The XRD measurements were performed by PROTO iXRD COMBO diffractometer with $\omega$-goniometer and $\{211\}$ diffraction line of $\alpha$-Fe was measured by $\operatorname{Cr} K \alpha$ radiation. Deformation of interplanar distances of variously rotated planes was converted to RS using the generalized Hooke's law according to the $\sin ^{2} \psi$ method. The corresponding neutron measurements were carried out on the diffractometer SPN-100 of the Nuclear Physics Institute which is installed at the channel HC-4 of the research reactor LVR-15 and operates at the neutron wavelength of $0.235 \mathrm{~nm}$. The SteCa software [5] was used to extract and analyse diffraction patterns from the recorded area detector data. The diffraction peak $\{110\}$ of $\alpha$-Fe was measured. The strain was calculated using the Hooke's law based on the angular deviation of the diffraction profile position from the value related to the stress-free sample. Full widths of the measured diffraction lines at half of the maximum ( $F W H M)$ were also evaluated for both methods. FWHM parameter depends on microstrains, crystallite size, density of dislocations and weight proportion of the hard phases.

Sample orientations during XRD and neutron diffraction are shown in Figs. 2a and 2b. The direction of the arrows always indicates the direction of the measured RS. The rotation of measured planes during $\sin ^{2} \psi$ measurements is also shown in Fig. $2 \mathrm{a}$ for XRD. Equivalent direction are therefore L' for $\mathrm{XRD}$ and $\mathrm{T}$ for neutron diffracton. The measurements were performed in three lines perpendicular to the welds. One line passes through the centre of the weld and the other two are located three millimetres below each surface, see red lines in Fig. 2c Upper side of the plate is the site which was firstly welded. To determine the real structure and RS by XRD on the cross section, it was necessary to cut the plate in two parts (solid lines and dashed lines in Fig. 2c) and the affected surface layer electrochemically etched.

\section{EXPERIMENT}

Gradients of RS measured by XRD in two directions on the surface of welded plates with a depth of penetration of the used radiation into the sample approx.

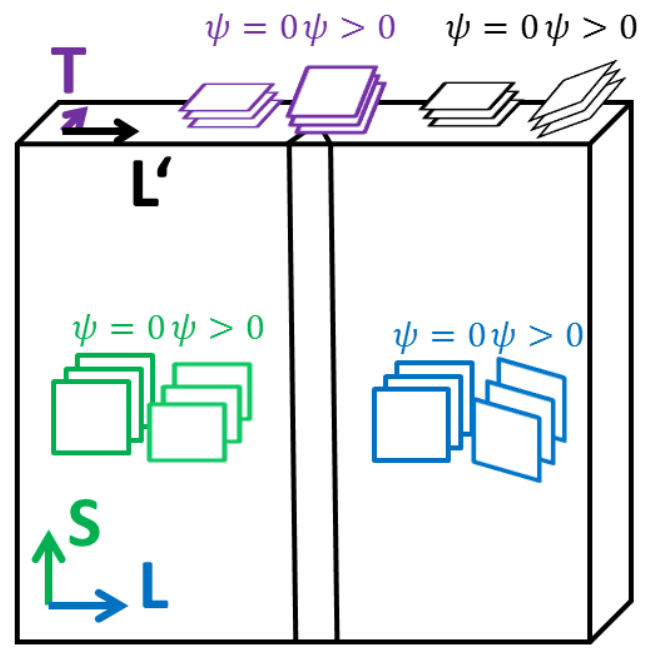

(A) . Sample orientations during XRD.

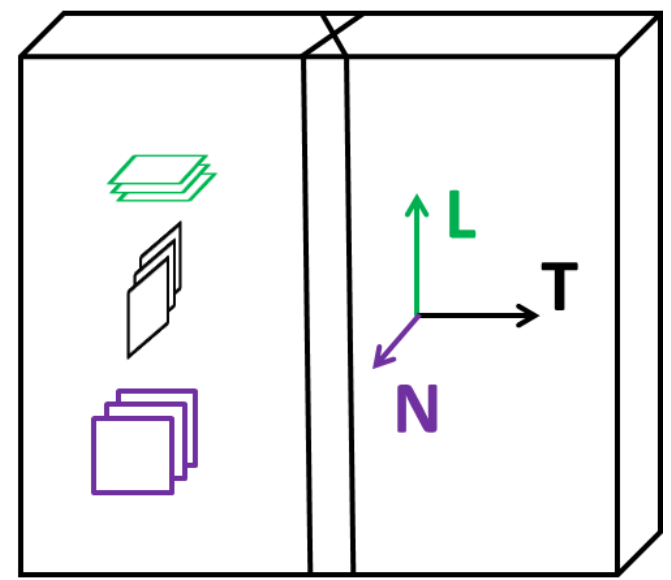

(B) . Sample orientations during neutron diffraction.

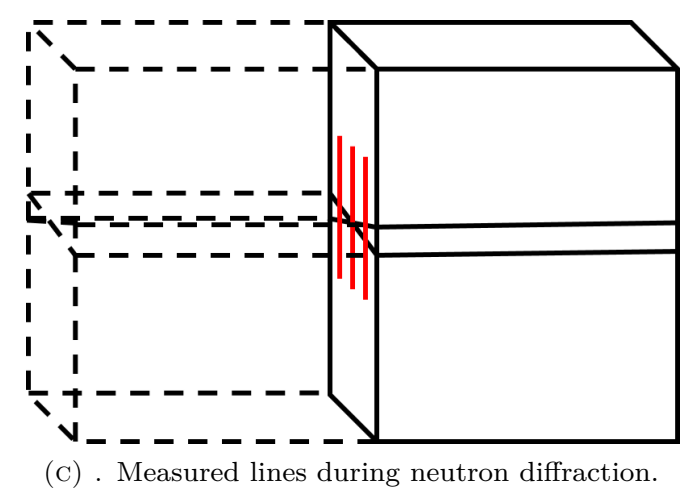

FiguRE 2. Sample orientations during experimental methods.

$4 \mu \mathrm{m}$ are plotted in Fig. 3. Dependences of threedimensional state of RS determined by neutron diffraction technique related to the middle line and two lines with depth of three millimetres below both surfaces of laser and MAG samples can be seen in Figs. 4 and 5 . The comparison of RS obtained by XRD in direction $\mathrm{L}$ ' and neutron diffraction in direction $\mathrm{T}$ is plotted in Figs. 6 and 7. The comparison of FWHM obtained 

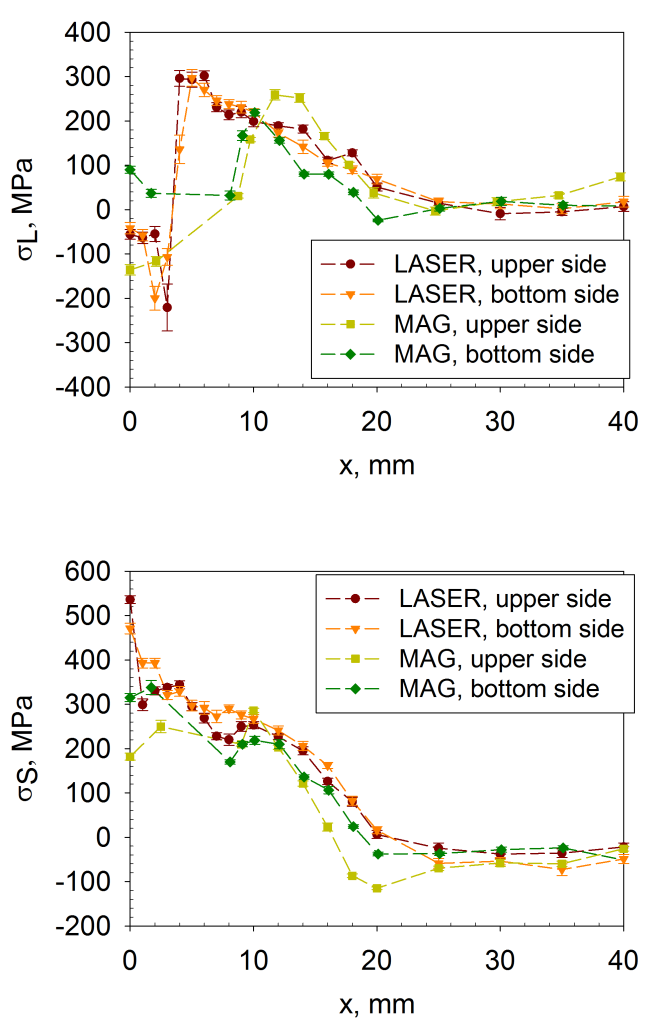

FiguRE 3. Surface residual stresses measured by XRD.

by XRD in direction L' and neutron diffraction in direction $\mathrm{T}$ is shown in Figs. 8 and 9 The parameter $\mathrm{x}$ is the perpendicular distance from the axis of the weld in all graphs.

The most significant tensile RS on the surface are in the direction $\mathrm{S}$ according to the predictions (see Fig. 1 and 3 for both samples. Tensile RS of the laser weld in the direction $\mathrm{S}$ exceed the yield strength of the used steel and for upper side even the tensile strength. This would suggest that the rapid cooling of laser weld created non-equilibrium hard phases with higher strength [6]. In $\mathrm{L}$ direction differently wide HAZ could be well observed.

According to Figs. 4 and 5 tensile RS within all the three measured lines obtained by neutron diffraction have the maximum in the longitudinal direction for both samples. RS in the laser weld at middle line exceeding $300 \mathrm{MPa}$ and for MAG in bottom line only $230 \mathrm{MPa}$. In the middle of the laser and MAG weld, the distribution of $\mathrm{RS}$ in both remaining directions $\mathrm{N}$ and $\mathrm{T}$ is the same. Shrinkage and other effects caused that RS were created in both directions homogeneously.

In the middle of the laser weld according to Figs. 6 and 7. RS obtained by neutron diffraction in the perpendicular direction to the weld reported more tension character than by XRD. This is most probably due to a significant contraction in the direction parallel to the weld. On the other hand in the case of the MAG weld, there are compressive stresses. This could suggest a predominance of phase transformation during the formation of RS rather than shrinkage. For both welds, RS obtained by XRD reported higher compressive results than obtained by neutron diffraction. The only exception is the upper line for the MAG weld, see Fig 7. Laser weld (according to the Fig. 4) has a higher tensile residual stresses along the weld, therefore, redistribution after cutting the sample probably produced the greatest compressive stresses measured by XRD. After cutting the plate, the weld probably slightly dropped, and thus it caused compressive RS in the plane perpendicular to the weld.

The comparison of the parameter FWHM using both methods exhibits in nearly all cases the same pattern, see Figs. 8 and 9 . Change in the parameter FWHM for MAG sample, which can be correlated with a width of HAZ, is recorded at a greater distance than for the laser weld. FWHM is in all cases for both methods higher for the laser sample, this would indicate a higher microstrain, density of dislocations and a greater amount of hard phases.

\section{Conclusions}

It was found from the residual stresses measured by neutron diffraction in three different sample orientations and in three different depths under the surface that the greatest gradient of residual stresses is in the direction parallel to the weld. According to the literature study and our results, greater tensile residual stresses exhibits the laser weld in almost all measured lines. Furthermore, the results describing HAZ width (changes in values of RS and FWHM) are very similar for both analytical methods (XRD and neutron diffraction). Both diffraction methods are able to complement each other.

Furthermore, the resulting residual stresses gradients obtained using both methods with a different approach are in certain correlation. Each method (XRD and neutron diffraction) describes the outcome of residual stresses in different context. The redistribution of RS during the cutting has not yet been accurately described, so further measurements and verification using mathematical modelling will be needed. We are currently performing the validation of measurement on the second MAG sheet.

\section{ACKNOWLEDGEMENTS}

Measurements were carried out at the CANAM infrastructure of the NPI ASCR Řež supported through MŠMT project No. LM2011019 and Czech Science Foundation GAČR through the project No. 14-36566G entitled as Multidisciplinary Research Centre for Advanced Materials. This work was supported by the Student Grant Competition CTU in Prague grant No. SGS16/245/OHK4/3T/14. The authors thank to MATEX PM for supplying the laser weld. 


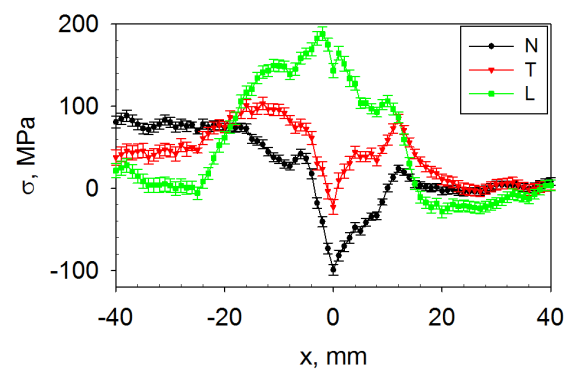

(A) . Laser - upper line.

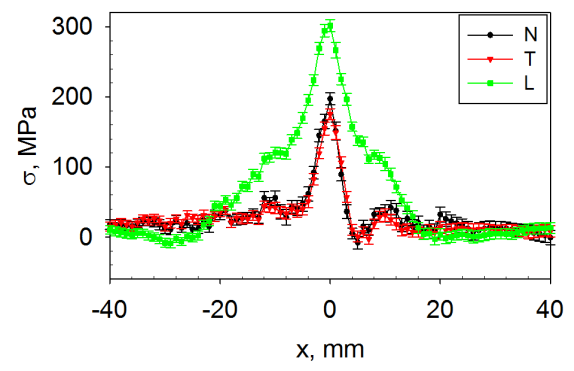

(в) . Laser - middle line.

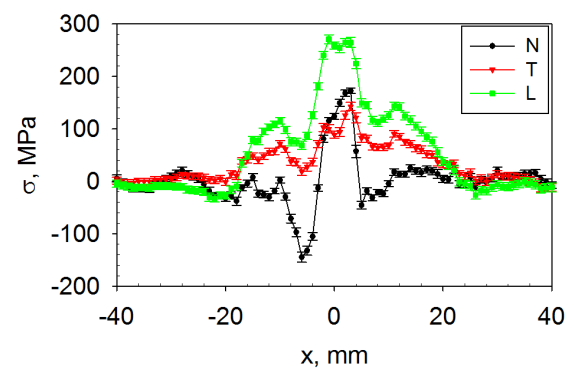

(C) . Laser - bottom line.

FiguRE 4. RS for laser sample along three measured lines obtained by neutron diffraction.

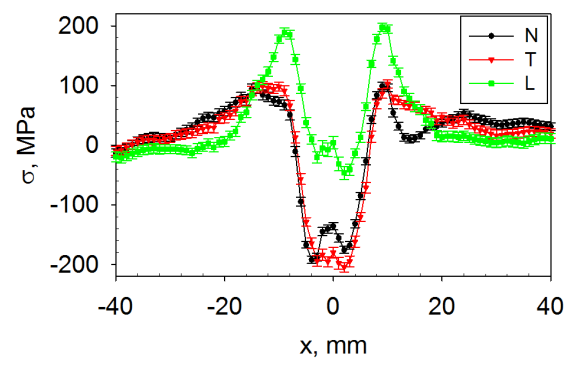

(A) . MAG - upper line.

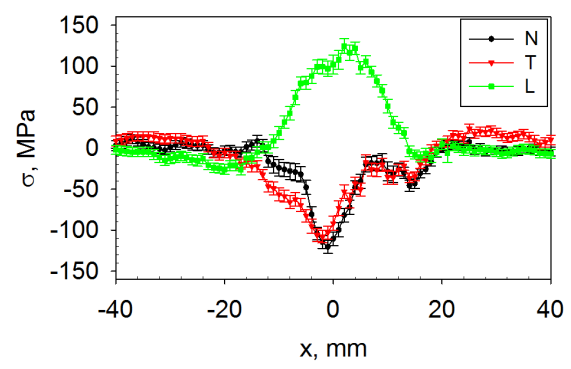

(в) . MAG - middle line.

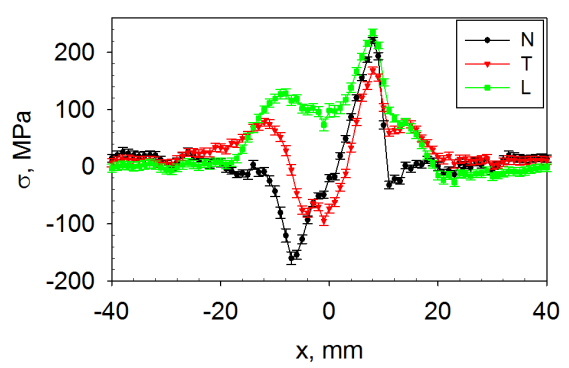

(c) . MAG - bottom line.

FIgURE 5. RS for MAG sample along three measured lines obtained by neutron diffraction. 


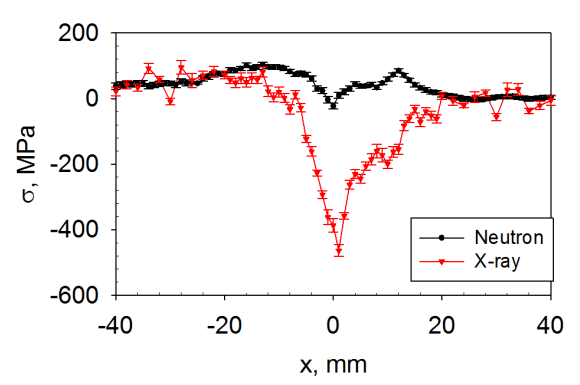

(A) . Laser - upper line.

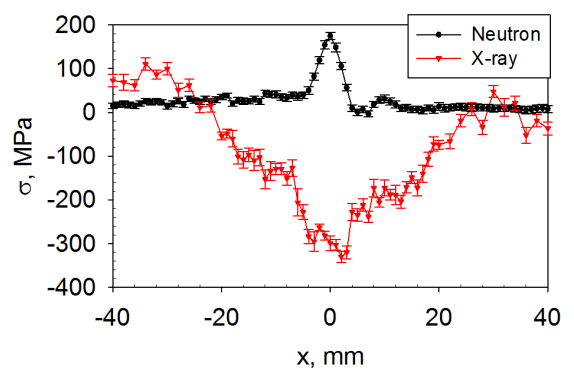

(в) . Laser - middle line.

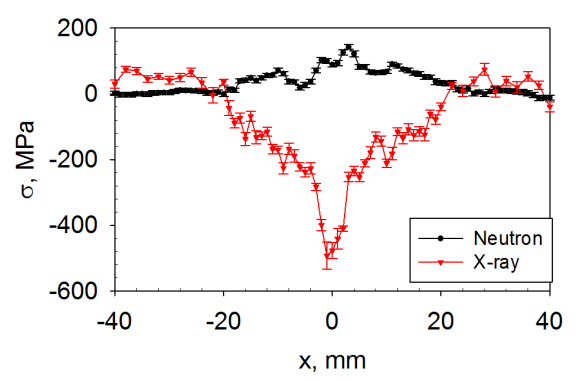

(c) . Laser - bottom line.

Figure 6. Comparison of RS obtained by XRD in direction L' and neutron diffraction in direction $\mathrm{T}$ along three measured lines for laser sample.

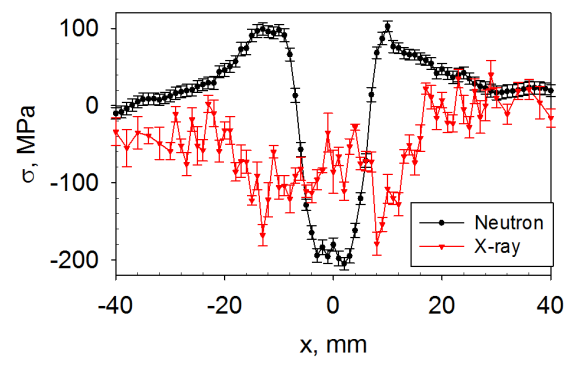

(A) . MAG - upper line.

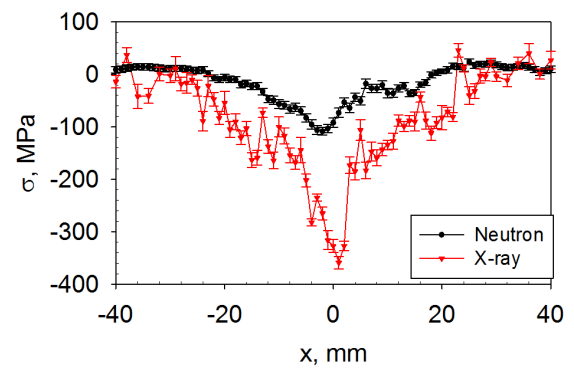

(в) . MAG - middle line.

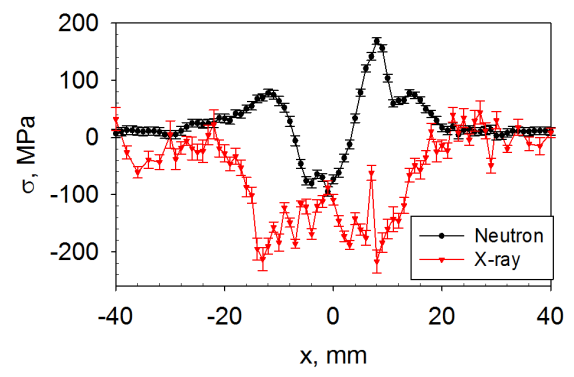

(c). MAG - bottom line.

Figure 7. Comparison of RS obtained by XRD in direction L' and neutron diffraction in direction $\mathrm{T}$ along three measured lines for MAG sample. 


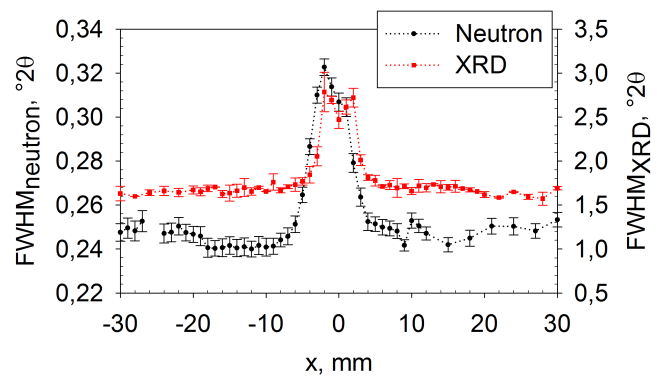

(A) . Laser - upper line.

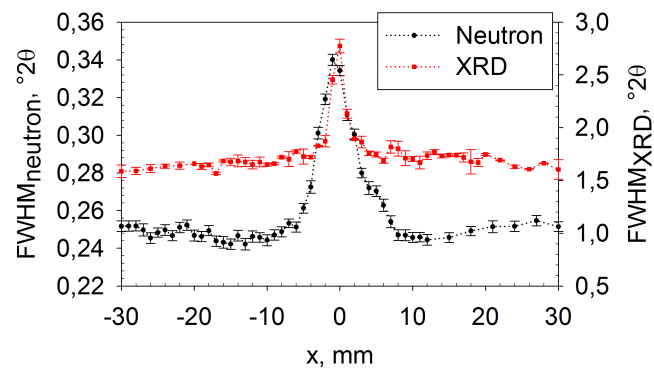

(B) . Laser - middle line.

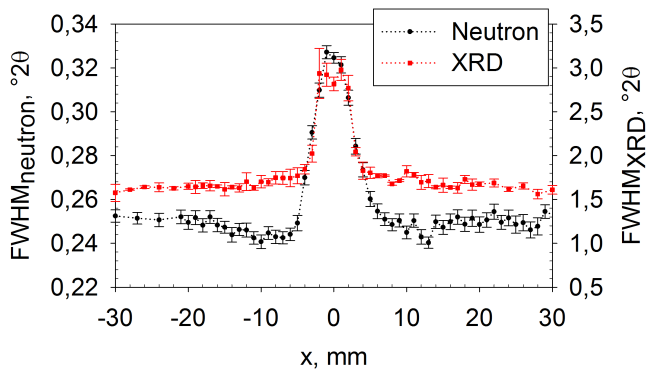

(C) . Laser - bottom line.

Figure 8. Comparison of $F W H M$ obtained by XRD in direction $\mathrm{L}^{\prime}$ and neutron diffraction in direction $\mathrm{T}$ along three measured lines for laser sample.

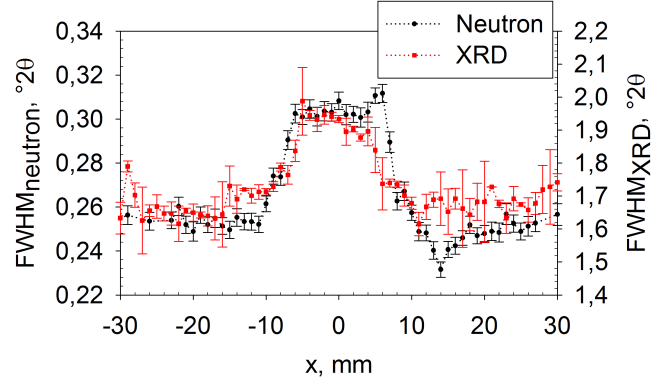

(A). MAG - upper line.

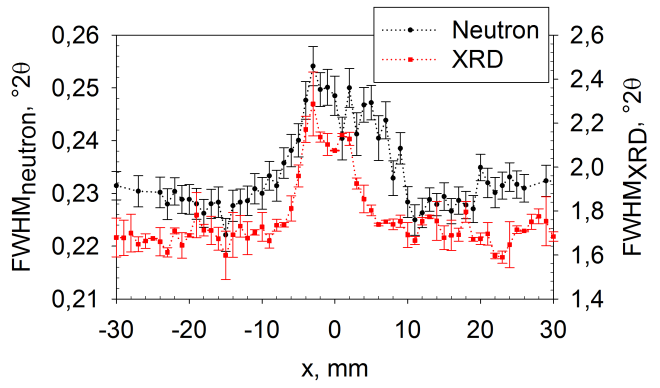

(в) . MAG — middle line.

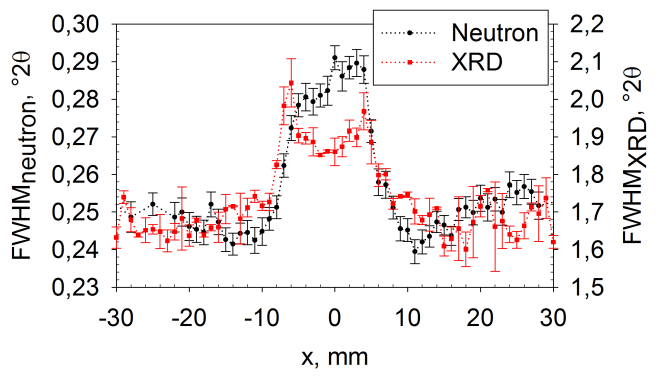

(c) . MAG - bottom line.

FIgURE 9. Comparison of $F W H M$ obtained by XRD in direction $\mathrm{L}^{\prime}$ and neutron diffraction in direction $\mathrm{T}$ along three measured lines for MAG sample. 


\section{REFERENCES}

[1] T. Nitschke-Pagel, K. Digler. Sources and consequences of residual stresses due to welding. Mater Sci Forum pp. 2777-2785, 2014. DOI:10.4028/www.scientific.net/MSF.783-786.2777

[2] M. Sokolov, A. Salminen, M. Kuznetsov. Laser welding and weld hardness analysis of thick section s355 structural steel. Mater Design 32(10):5127-5131, 2011. DOI:10.1016/j.matdes.2011.05.053

[3] C. Lachmann, T. H. Nitschke-Pagel, H. Wohlfahrt. Nondestructive characterization of residual stress relaxation and fatigue processes in cyclically loaded welded joints. pp. 1400-1409. Oxford, UK, 2000.
[4] W. Woo, et al. Neutron diffraction measurements of residual stresses in a $50 \mathrm{~mm}$ thick weld. Mater Sci Eng A 528(12):4120-4124, 2011. DOI:10.1016/j.msea.2011.02.009

[5] C. Randau, U. Garbe, H. G. Brokmeier. Stresstexturecalculator: a software tool to extract texture, strain and microstructure information from area-detector measurements. J Appl Cryst 44(3):641-646, 2011. DOI:10.1107/S0021889811012064

[6] N. Jia, et al. An in situ high-energy x-ray diffraction study of micromechanical behavior of multiple phases in advanced high-strength steels. Acta Mater 57(13):39653977, 2009. DOI:10.1016/j.actamat.2009.05.002 